\title{
Enhanced analgesic cholinergic tone after neuropathy
}

2

3 Dhanasak Dhanasobhon ${ }^{1,2}$, Maria-Carmen Medrano ${ }^{1,2}$, Yunuen Moreno-Lopez ${ }^{1,2}$, Sehrazat

4 Kavraal $^{1,2}$, Charlotte Bichara ${ }^{1,2}$, Rémy Schlichter ${ }^{1,2}$, Perrine Inquimbert ${ }^{1,2}$, Ipek Yalcin ${ }^{1,2}$, and

5 Matilde Cordero-Erausquin ${ }^{1,2,3 *}$

$6 \quad{ }^{1}$ Institut des Neurosciences Cellulaires et Intégratives, Centre National de la Recherche

7 Scientifique, 67000 Strasbourg, France

$8 \quad$ 2Université de Strasbourg, Strasbourg, France

$9 \quad{ }^{3}$ University of Strasbourg Institute for Advanced Study (USIAS), Strasbourg, France

10 Number of pages: 24

11 Number of figures: 8

12 *Corresponding author: Matilde Cordero-Erausquin, PhD, HDR, CR1 CNRS

13 Institut des Neurosciences Cellulaires et Intégratives - CNRS UPR 3212

148 allée du Général Rouvillois, 67000 Strasbourg cedex, France

15 Tel: (+33) 3884566 60/ Fax: (+33) 388601664

16 e-mail : cordero@unistra.fr 


\section{Abstract}

19 At the spinal cord level, a tone of endogenous acetylcholine (ACh) modulates nociceptive sensory 20 processing. Increasing the level of spinal ACh induces analgesia in naïve animals or in situation of 21 acute pain in the clinics, but whether this is still the case in situations or models of chronic pain is 22 controversial. Here, we demonstrate the persistence, and even increased impact of the analgesic 23 cholinergic tone acting through nicotinic receptors in neuropathic mice. The neuropathy does not 24 affect the number and properties of dorsal horn cholinergic neurons, proposed to be the source of 25 spinal ACh. Subthreshold doses of acetylcholinesterase (AChE) inhibitors in sham animals become 26 anti-allodynic in cuff mice suggesting that the alterations occur in the cholino-receptive neurons. Thus 27 endogenous cholinergic signaling can be manipulated with low doses of drugs to relieve mechanical 28 allodynia in animal neuropathy models. This opens new avenues with potentially fewer side effects for 29 neuropathic pain treatment.

30 Key words: behavior, cholinergic system, dorsal horn, electrophysiology, mechanical stimuli, mice, 31 neuropathic pain, nicotinic receptors, spinal cord, von-Frey test 


\section{Introduction}

Endogenous acetylcholine (ACh) is an important modulator of nociceptive sensory processing in the spinal cord. Epidural administration of neostigmine, an acetylcholinesterase (AChE) inhibitor that induces an increase in ACh spinal level by preventing its degradation, produces pain relief for child birth and post-operation pain in clinics (Eisenach, 2009). In rodents, local spinal injection of AChE inhibitors (by intrathecal injections, or i.t.) similarly produces analgesia to thermal or chemical noxious stimulation (Chen \& Pan, 2003; Chiari, Tobin, Pan, Hood, \& Eisenach, 1999; Hartvig, Gillberg, Gordh, \& Post, 1989; Miranda, Sierralta, \& Pinardi, 2002; Naguib \& Yaksh, 1994). The analgesic effect of endogenous ACh is not only observed when its level is artificially increased (by AChE antagonists), but also with its physiological levels. Indeed, impairment of cholinergic signaling by locally antagonizing ACh receptors (nicotinic or muscarinic) or by knocking-down the $\beta 2$ subunit of nAChRs induces hyperalgesia and/or allodynia in rodents (Hama \& Menzaghi, 2001; Rashid \& Ueda, 2002; Yalcin, Charlet, et al., 2011; Zhuo \& Gebhart, 1991). This suggests that a basal “tone” of spinal ACh modulates the nociceptive threshold.

A dense plexus of cholinergic processes (composed of both dendrites and axons) lies in laminae (L) II-III of the dorsal horn (DH) of rodents (Barber, et al., 1984; Mesnage, et al., 2011). Within this plexus, cholinergic dendrites receive synaptic contacts from primary afferents, and cholinergic axons make presynaptic contacts onto them (Olave, Puri, Kerr, \& Maxwell, 2002; Ribeiro-da-Silva, 2004), offering a potential substrate for sensory modulation. In rodents, sparse cholinergic neurons with extended ramification, are located in lamina III-IV and have been proposed to be the source of this plexus. They could therefore be responsible for cholinergic analgesia (Mesnage, et al., 2011). Interestingly, we have demonstrated that a similar cholinergic population exists in the dorsal horn of macaque monkeys, with comparable localization, density and most importantly, synaptology (Pawlowski, et al., 2013). The behavioral and anatomical similarities have confirmed that mice are a valuable model to study cholinergic analgesia. But how this population of cholinergic interneurons ultimately impacts nociceptive behavior is still unknown.

AChE inhibitors are used in the clinics only in a context of acute pain. Yet chronic pain is also a devastating and widespread problem, inadequately treated according to two-thirds of chronic pain sufferers (Breivik, Collett, Ventafridda, Cohen, \& Gallacher, 2006). How the endogenous spinal cholinergic control of analgesia evolves in situations of chronic pain in primate is unknown, and data on rodents are scarce and at times contradictory. While Rashid and Ueda have proposed that the cholinergic tone is disrupted in neuropathic animals as shown by the loss of effect of the i.t. injection of a nicotinic antagonist (Rashid \& Ueda, 2002), i.t. injections of AChE antagonists still have an analgesic effect (Hwang, et al., 1999; P. Lavand'homme, Pan, \& Eisenach, 1998; P. M. 
68 locally available to affect sensory processing. The dorsal horn of the spinal cord is a site for numerous

69 forms of plasticity occurring during chronic pain (Tsuda, Koga, Chen, \& Zhuo, 2017), and it was

70 recently demonstrated that synaptic inputs or intrinsic properties of several interneuronal populations

71 are altered during the neuropathy (Cheng, et al., 2017; Imlach, Bhola, Mohammadi, \& Christie, 2016;

72 Peirs, et al., 2015; Petitjean, et al., 2015). How the cholinergic population, and more generally the

73 spinal cholinergic analgesia, is affected in chronic pain models remained to be evaluated and was the

74 main goal of this study. Accordingly, we have combined behavioral, histological, in vivo and in vitro

75 electrophysiological recordings to improve our understanding of spinal cholinergic analgesia. We

76 specifically addressed the question of its plasticity in conditions of chronic pain, and of potential

77 underlying mechanisms. 


\section{Results}

2.1 Behavioral effect of cholinergic modulation onto mechanical responses

We first characterized the dose- dependent effect of spinal cholinergic modulation onto transmission of mechanical information in naïve CD1 mice. For this purpose, the mechanical paw withdrawal threshold (PWT) was evaluated with the von Frey test, before and after i.t. injection of the AChE inhibitor physostigmine. Physostigmine (15 nmol, i.t), produced a long-lasting increase in the mechanical withdrawal threshold (Fig. 1A, Suppl. Fig. 1; statistics are presented in the figure legends) while lower doses (1.5-7.5 nmol) were without effect. This indicates that ACh is endogenously released and its level in the spinal cord closely controlled by AChE. We thus confirm that, if its concentration is increased (by inhibiting its hydrolysis), ACh reaches downstream targets, activating a spinal circuit leading to analgesia.

In order to investigate whether this cholinergic analgesic mechanism is active in baseline physiological conditions, we interfered with it by blocking cholinergic nicotinic receptors. We thus injected the non-specific nicotinic antagonist mecamylamine (10-400 nmol, i.t.) and tested at different time points after the injection (Fig.1B). Mecamylamine induced a dose- and time-dependent reduction in the PWT: for all doses, the PWT was statistically reduced as soon as 15 min post-injection, and for all but the $10 \mathrm{nmol}$ dose, the effect lasted for more than two hours. In the 15 min - 2 hours time window, there was no significant difference between the three higher doses (at each time point, 400 nmol or 100 nmol vs. $20 \mathrm{nmol}: \mathrm{p}>0.99$, Bonferroni's multiple comparisons test) suggesting that the effect already plateaued with $20 \mathrm{nmol}$ mecamylamine. 150 minutes after the injection, however, animal that had received $20 \mathrm{nmol}$ of mecamylamine had a PWT statistically different from the one of animals that had received 100 or $400 \mathrm{nmol}$ mecamylamine (20 nmol vs. $100 \mathrm{nmol}$, p=0.0007; $20 \mathrm{nmol}$ vs. $400 \mathrm{nmol}, \mathrm{p}=0.0001$, Bonferroni's multiple comparisons test). Indeed, the allodynia evoked by 10 nmol mecamylamine had faded away after 150 minutes, while it was still present in the 20, 100 and 400 nmol injection groups (comparisons with saline presented in Fig. 1B). This suggests that the cholinergic analgesic mechanism is active in baseline physiological conditions, either because ACh is continuously released and activating downstream circuits, or because it is released as a consequence of the mechanical stimulation. Whatever the underlying cause, the pharmacological effect of a nicotinic antagonist defines the presence of an analgesic spinal cholinergic tone, acting via nicotinic receptors.

High concentrations of mecamylamine are known to have potentially non-specific effects (McDonough \& Shih, 1995; O'Dell \& Christensen, 1988). We used $\beta 2$ nicotinic knock-out mice ( $\beta 2$ $\mathrm{KO}$ ) to verify whether the highest dose of mecamylamine used (400 nmol) was acting specifically through inhibition of $\mathrm{nAChR}$ receptors. While $400 \mathrm{nmol}$ i.t. mecamylamine induced a marked reduction of the PWT in WT animals, it produced no effect on $\beta 2 \mathrm{KO}$ mice (Fig. 1C, $400 \mathrm{nmol}$ 
mecamylamine vs saline in WT mice: $\mathrm{p}=0.0042$, and in KO: $\mathrm{p}>0.99$, Bonferroni's multiple comparisons test). Moreover, we observed that this high concentration of mecamylamine did not affect motor- coordination as evaluated by the rotarod test in naive mice (Fig. 1D), suggesting that the observed modulation strictly involved mechanical sensory processing.

\subsection{The spinal cholinergic tone is present after neuropathy}

We then investigated the alteration of this analgesic cholinergic tone in a mouse model of neuropathy. We used the cuff model and analyzed the effect of i.t. injection of mecamylamine (10-400 nmol) on the PWT of sham and cuff mice. For the ipsilateral, but not the contralateral, paw there was a significant interaction between Surgery, Time and Drug $(\mathrm{p}=0.0354)$, indicating that the neuropathy modified the response of the ipsilateral paw to mecamylamine.

Mecamylamine induced a dose-dependent reduction in the mechanical withdrawal threshold of the contralateral hindpaw in sham and cuff mice (Fig. 2A Top, in sham, for all doses of mecamylamine vs. saline at $15 \mathrm{~min}, \mathrm{p}<0.0001$ in post-hoc tests; in cuff, similar comparison yielded to $\mathrm{p}<0.0001$ for the 20 nmol, $100 \mathrm{nmol}$ and $400 \mathrm{nmol}$ mecamylamine doses, and $\mathrm{p}=0.0257$ for the $10 \mathrm{nmol}$ dose, Bonferroni's multiple comparisons test). For the ipsilateral hindpaw, 10 and $20 \mathrm{nmol}$ was ineffective in cuff as opposed to sham groups (Fig. 2A Bottom, post-hoc tests at $15 \mathrm{~min}$, drug vs. saline: $\mathrm{p}=$ $<0.0001$ for 10 and $20 \mathrm{nmol}$ mecamylamine in sham and respectively p>0.99 and $\mathrm{p}=0.159$ in cuff; Bonferroni's multiple comparisons test). The mechanical allodynia observed in cuff animals was exacerbated when higher doses were injected (Fig. 2A; post-hoc tests at $15 \mathrm{~min}$, in cuff mice: for 100 nmol mecamylamine vs. saline, $p=0,0017$ and for 400 nmol mecamylamine vs. saline, $p<0.0001$; Bonferroni's multiple comparisons test). Analysis of the dose-response curve demonstrates a shift to the right in cuff mice, with higher doses being necessary to obtain a significant effect (Fig. 2B; Ipsilateral side - Least square fit, $\mathrm{p}<0.001$ ). Thus the spinal cholinergic tone is still present in cuff animals, involving nAChRs and affecting mechanical responses. The shift to the right of the mecamylamine dose-response curves further suggests that the cholinergic tone might be increased by the pathology, either because more ACh is released or because endogenous ACh has an increased effect on its post-synaptic targets. We next investigated these two possibilities.

\subsection{Plasticity of LIII/IV cholinergic interneurons during neuropathy}

Lamina III-IV cholinergic neurons are the most plausible source of the spinal cholinergic tone. We thus closely analyzed this population in sham and neuropathic mice to test the hypothesis that the release of ACh could be increased following the neuropathy. In particular, we assessed 3 different criteria: (1) synaptic inputs, (2) active and passive electrophysiological properties, and (3) number of cholinergic interneurons. 
We then assessed whether DH cholinergic neurons could be differentially activated in sham vs. cuff by comparing the synaptic inputs received by these neurons in these two conditions. Cholinergic interneurons are located in lamina III-IV, and best preserved in horizontal sections (Mesnage, et al., 2011). However, we first performed a set of recordings of lamina II neurons in transverse slices, in order to put our results in context with the literature, mostly focusing on this layer. Indeed, while the cuff model of mouse peripheral neuropathy has been well described in behavioral terms (Benbouzid, et al., 2008; Yalcin, Bohren, et al., 2011), we were the first to analyze the plasticity occurring in the spinal cord with electrophysiology in vivo (Medrano, Dhanasobhon, Yalcin, Schlichter, \& CorderoErausquin, 2016) and now in vitro. Recordings were performed in the ipsilateral (to cuff insertion) DH in the presence of $0.5 \mu \mathrm{M}$ TTX (Fig. 3A). The frequency of miniature IPSCs was lower in cuff mice compared to sham controls, whereas the frequency of miniature EPSCs was unchanged (Fig. 3B). The amplitude for miniature currents (either EPSC or IPSCs) were similar for sham and cuff mice (Fig. 3B). Thus only the frequency of mIPSCs is modified in lamina II neurons in cuff animals, similarly to what is observed in the spared nerve or chronic constriction injury models (Iura, Takahashi, Hakata, Mashimo, \& Fujino, 2016; Moore, et al., 2002).

We then moved to lamina III-IV and to horizontal slices to record spontaneous and miniature EPSCs and IPSCs from ChAT::EGFP and non-EGFP (unidentified) neurons in their close vicinity in sham and cuff mice (Fig. 4A, Suppl. Fig. 2). For EPSCs, there were no significant difference between the groups. For IPSCs, there was on average more inhibition in cuff vs. sham mice (not specific to any neuronal population under study, or to any type of current, spontaneous vs. miniatures), and cholinergic neurons had on average less inhibitory inputs than non-cholinergic ones (whether in sham or in cuff mice). There was therefore no change of inputs frequency that was specific to cholinergic neurons after neuropathy (Fig. 4B). We compared with a similar approach the amplitude of miniature EPSC and IPSC in the different neurons and animals, and observed no significant difference between groups (Fig. 4C).

172 These recordings reveal an increase in the frequency of inhibitory inputs that is not specific to lamina

173 III-IV cholinergic neurons as it is also observed in non-EGFP neurons in the same laminae, while 174 lamina II neurons experience a decrease of the frequency of IPSCs. The increased frequency of inhibitory currents would argue, if anything, that cholinergic neurons are less prone to firing (and

176 releasing $\mathrm{ACh}$ ) in neuropathic conditions. In order to test this hypothesis, we next compared the 177 capacity of cholinergic neurons to be excited by direct depolarization in sham vs. cuff mice. 
We analyzed the firing patterns of recorded neurons after injection of depolarizing currents, and classified their discharge profiles as either "tonic", "single-spike”, or "phasic" (cf. Methods, Fig. 5A1).There was no statistical difference between ChAT::EGFP and non-EGFP neurons concerning the proportion of the different firing patterns in the two different animal groups (Fig. 5B). A fraction of recorded neurons presented spontaneous ongoing firing at rest (rheobase of 0pA, Fig. 5C) but their proportion was not different between types of neurons (ChAT::EGFP vs. non-EGFP) or type of surgery (sham vs. cuff) ( $3 \times 3 \times 3$ contingency table, $p=0.2674)$. Specifically, 2 out of 6 ChAT::EGFP neurons (33.3\%) were spontaneously active in sham mice, but none out of $8(0 \%)$ in cuff, while 1 out of 7 non-ChAT neurons (14.2\%) was spontaneously active in sham mice vs. 2 out 6 (33.3\%) in cuff mice. There also was no statistical difference in the rheobase in the different groups (Fig. 5C). We compared the input-output properties of the different neurons (measured as mean instantaneous firing frequency induced by an increasing depolarizing current, Fig. 5D). On average, there was no effect of the surgery on the instantaneous firing frequencies, but there was an effect of the intensity of the injected current, as well as of the type of neurons: ChAT::EGFP vs. non-EGFP neurons (Suppl. Fig. 3). Moreover, there was an interaction between the response to different injected currents and the type of neurons, as non-EGFP neurons fired more than ChAT::EGFP neurons when large currents were injected (120 to $180 \mathrm{pA}$, suppl. Fig. 3). There was no interaction of these factors with the surgery, suggesting that the input-output properties of the neurons were not affected by the surgery. We also investigated the passive membrane properties of recorded neurons. There was no difference among groups (ChAT::EGFP or non-EGFP neurons, sham or cuff) in the resting potential (Fig. 5C), the input resistance (Fig 5C) or the amplitude of a sag current (Fig. 5A2, 5E).

Altogether, these measures indicate that the network plasticity observed in slices obtained from cuff mice does not alter the excitability of cholinergic neurons.

\subsubsection{Number of cholinergic interneurons}

There is an on-going debate on whether there is a loss of neurons in the DH that would contribute to the development of hyperalgesia or allodynia following neuropathy. Therefore, we investigated whether DH cholinergic interneurons were affected. We have previously shown that ChAT::EGFP mice are a good model for studying DH cholinergic neurons (Mesnage, et al., 2011). We confirm here that the vast majority (98\%) of lamina III-IV EGFP+ are ChAT immunoreactive. We compared the number of EGFP+ neurons in the contralateral and ipsilateral DH of adult ChAT::EGFP+ mice two weeks after surgery. All sections from L3 - L6 spinal segments were taken into account (Fig. 6). On the ipsilateral dorsal horn, there was no statistical difference between the number of ChAT::EGFP+ neurons per $40 \mu \mathrm{m}$ thick sections in sham (on average $2.13 \pm 0.38$ neurons) vs. cuff $(2.07 \pm 0.38$ neurons). This ruled out a change in the number of $\mathrm{DH}$ cholinergic neurons after neuropathy. 
As we found no evidence of specific increased excitation or excitability of DH cholinergic neurons in neuropathic animals, nor a change in neuronal density, we next asked whether the increased impact of the cholinergic tone in these animals could be explained by modifications in direct or indirect targets of cholinergic neurons, i.e. downstream of the tone itself. The cholinergic tone has never yet been described in in vivo electrophysiological recordings, nor its consequences on the response of DH neurons to tactile stimulation. We thus first studied these aspects by recording DH neurons in vivo in sham and cuff animals to identify a potential substrate to the antinociceptive cholinergic tone and its plasticity, and then turned to pharmacological behavioral experiments. animals within the second and third week after surgery. Neurons were selected for their localization in the DH (Fig. 7A) and their response to mechanical stimulation of the hindpaw, either non-nociceptive (touch) and/or nociceptive (pinch) (see methods and (Medrano, et al., 2016)). Local application of 100 $\mu \mathrm{M}$ mecamylamine (on the spinal cord) induced an increased response to mechanical stimulation in neurons recorded from both sham and cuff mice (Fig. 7B). The recorded neurons spanned many DH laminae (Fig. 7A) and the response amplitudes showed high variability, precluding the identification of a potential differential effect of mecamylamine in sham vs. cuff mice. These recordings however demonstrate for the first time in vivo the presence of a spinal cholinergic tone, acting through nicotinic receptors, attenuating mechanical responses. This could be a substrate for the antinociceptive spinal cholinergic tone observed in behavioral experiments.

\subsubsection{Effect of an AChE inhibitor in neuropathic animals}

235 In order to evaluate a potential change of the network downstream of the cholinergic tone, we turned 236 back to behavioral experiments and compared the effect of endogenous spinal ACh in sham and cuff 237 mice by i.t. injection an AChE inhibitor. Physostigmine effect on the contralateral paw was not 238 affected by the surgery (Fig. 8A). For the ipsilateral paw in contrast, the surgery modified the response 239 to physostigmine: in neuropathic mice, $7.5 \mathrm{nmol}$ physostigmine injected i.t. was able to alleviate the mechanical allodynia of the ipsilateral paw while it was ineffective to increase PWT in sham animals (Fig. 8A, at 15 min: 7.5 nmol physostigmine vs. saline $\mathrm{p}<0.0001$ in cuff and $\mathrm{p}>0.99$ in sham;

242 Bonferroni's multiple comparisons test). The $15 \mathrm{nmol}$ dose however was analgesic for both paws in 243 both sham and cuff mice (Fig. 8A, 15 min: drug vs. saline $\mathrm{p}<0.0001$ for both sham and cuff; 244 Bonferroni's multiple comparisons test). The dose response curve demonstrates that physostigmine 245 differentially affected the relative mechanical response in the ipsilateral (but not contralateral) paw of 
bioRxiv preprint doi: https://doi.org/10.1101/2020.06.11.146852; this version posted June 12,2020 . The copyright holder for this preprint

(which was not certified by peer review) is the author/funder, who has granted bioRxiv a license to display the preprint in perpetuity. It is made available under aCC-BY-NC-ND 4.0 International license.

246 cuff vs. sham (Fig. 8B). This favors the hypothesis of a post-synaptic plasticity affecting the

247 downstream players of cholinergic transmission (i.e. post-synaptic receptors). 


\section{Discussion}

250 In this study, we performed an in depth analysis of the antinociceptive spinal cholinergic tone. We

251 demonstrated the persistence and even increased impact of this tone in a model of neuropathic pain,

252 and ruled out changes in lamina III-IV cholinergic neurons as a source of this plasticity. Instead, our

253 data show an increase in the sensitivity of downstream targets to spinal ACh. Low doses of AChE

254 inhibitors, without any effect in control mice, are analgesics in cuff mice which may open new

255 avenues for therapeutical relief of neuropathic pain conditions.

256 The existence of a "spinal cholinergic tone" has been proposed in the early 1990's and confirmed 257 since then (Rashid, Furue, Yoshimura, \& Ueda, 2006; Rashid \& Ueda, 2002; Zhuo \& Gebhart, 1991)

258 to describe an endogenous mechanism whose presence is revealed by a pharmacological effect of its 259 antagonization. Although the term is reminiscent of the word "tonic", it gives no inference on the way 260 ACh is released: either repetitively (through the "tonic firing” of a cholinergic neuron), or phasically 261 (upon a specific stimulation, e.g. nociceptive stimulation (Eisenach, Detweiler, Tong, D'Angelo, \& 262 Hood, 1996)). We here similarly define the "cholinergic tone" through the allodynic effect of i.t. 263 mecamylamine injection, suggesting that in basal conditions ACh has an ongoing (or provoked) 264 analgesic effect.

265 The cuff, a neuropathic pain model chosen for this study, was originally developed for rats (Mosconi 266 \& Kruger, 1996) and then adapted for mice (Benbouzid, et al., 2008). In a previous study, we 267 characterized by in vivo recordings the loss of inhibitory tone in the spinal DH in this model 268 (Medrano, et al., 2016). We provide here for the first time in vitro recordings in the spinal cord of 269 these mice and show that overall, the changes observed in synaptic inputs of DH lamina II neurons in 270 other models are similarly observed in the cuff model. Indeed, we observed a reduction in the 271 frequencies, but not amplitude, of miniature GABA-A currents in lamina II as reported in the spared 272 nerve injury model (SNI) and the chronic constriction injury (CCI) model (Iura, et al., 2016; Moore, et 273 al., 2002). However, we observed no change in the frequency for mEPSCs while a reduction is 274 reported in a study focusing on GABAergic interneurons in the CCI model (Leitner, et al., 2013). 275 Whether this difference is due to a difference in the model, or to the fact that the Leitner et al. study 276 focuses only on GABAergic neurons remains to be established. Importantly, all of our experiments 277 were conducted from the first up to the third week after cuff surgery, a time window where the 278 allodynia phenotype is robustly established but where anxiodepressive-like behaviors are not yet 279 present (Benbouzid, et al., 2008; Yalcin, Bohren, et al., 2011).

280 A previous study exploring the plasticity of the spinal cholinergic tone used the partial ligation of the 281 sciatic nerve model, and analyzed the thermal paw withdrawal (Rashid \& Ueda, 2002). They showed that i.t. injection of $10 \mathrm{nmol}$ mecamylamine had no effect in the injured mice, but higher doses were 
not tested. In the von Frey mechanical test, we similarly found that $10 \mathrm{nmol}$ mecamylamine was without effect but higher doses exacerbated the allodynia, demonstrating a shift to the right of the dose-response curve of the antagonist. Therefore, rather than a loss of cholinergic tone, our results suggest that its impact is actually increased by the neuropathy.

We also investigated the possible sources of this plasticity. Our previous studies concluded that spinal lamina III-IV cholinergic neurons were the main source of ACh at this level and that their morphology and projections were well suited to impact nociceptive processing, both in rodents and in primates (Mesnage, et al., 2011; Pawlowski, et al., 2013). These cholinergic interneurons are also GABAergic (Mesnage, et al., 2011; Miles, Hartley, Todd, \& Brownstone, 2007), and the existence of GABAergic cell death after peripheral neuropathy and its contribution to mechanical allodynia has been proposed although controversially debated (Inquimbert, et al.; Polgar, Gray, Riddell, \& Todd, 2004; Scholz, et al., 2005). We took advantage of the ChAT::EGFP mice that have been shown to be a reliable tool to study DH cholinergic neurons (Mesnage, et al., 2011), and found no difference in the number of ChAT::EGFP neurons following neuropathy. Nevertheless, other morphological alterations could exist, as it has been suggested for parvalbumin (PV) interneurons (Petitjean, et al., 2015). Indeed, their number remained unchanged after spared nerve injury in mice, but the number PV+ appositions on PKC-gamma cell bodies was reduced following injury. PV neurons provide feed-forward inhibition onto PKC-gamma neurons thus 'gating' the transmission of non-noxious information into nociceptive circuits. This decreased innervation has therefore been proposed to be a substrate of mechanical allodynia (Petitjean, et al., 2015).

Beyond changes in density or morphology, a modification of the synaptic inputs received by the cholinergic interneurons could explain a change in the spinal cholinergic tone. A combination of recent reports demonstrate that $A \beta$ inputs gain access to LI projection neurons through the loss of multiple feedforward inhibitory circuits following nerve injury (Abraira, et al., 2017; Alba-Delgado, et al., 2015; Cheng, et al., 2017; Foster, et al., 2015; Imlach, et al., 2016; Lu, et al., 2013; Peirs, et al., 2015; Petitjean, et al., 2015). Although the network organization of the dorsal horn starts to be elucidated (Cordero-Erausquin, Inquimbert, Schlichter, \& Hugel, 2016), the position of cholinergic neurons with respect to this feedforward inhibitory circuit is still unknown. In the cuff model, we observed a decrease in inhibition in lamina II neurons. Those lamina II neurons could be inhibitory neurons projecting to deeper laminae (Santos, Rebelo, Derkach, \& Safronov, 2007), and their 313 disinhibition could thus explain the increased frequency of inhibitory currents observed in laminae III-

314 IV. This increased inhibition of cholinergic interneurons does not translate into decreased excitability, 315 indicating that the release of ACh might not be significantly impaired. Overall, the membrane 316 properties of these neurons were unchanged in neuropathic animals. Similar observations are reported 
Forsthuber, \& Sandkuhler, 2013; Schoffnegger, Heinke, Sommer, \& Sandkuhler, 2006), suggesting that changes in membrane excitability or altered firing patterns in the spinal cord dorsal horn are unlikely causes for the alterations underlying neuropathic pain or for the observed plasticity of the cholinergic tone.

As the analysis of DH cholinergic interneurons did not explain the increased impact of the cholinergic tone in neuropathic animals, we then explored downstream mechanisms, in the spinal nociceptive network. Previous in vivo recordings performed in rats demonstrated that projection neurons in the cervical cord had an increased response to mechanical stimulation after topical application of an AChE inhibitor (neostigmine) (Chen \& Pan, 2004). Using similar recordings in mice, but on unidentified DH neurons, our study illustrates that a nicotinic antagonist has the opposite effect on mechanical responses, demonstrating for the first time a potential substrate the analgesic cholinergic tone in vivo. Our recordings also show that this tone is still present after cuff injury, but no differential effect of the cholinergic tone after neuropathy is revealed.

To finally identify the plasticity that could explain the increased impact of the cholinergic tone in cuff mice, we analyzed the analgesic properties of increasing i.t. doses of an AChE inhibitor, physostigmine, with the von Frey test. These experiments demonstrated a shift to the left of the dose response curve of this drug since doses that were subthreshold in sham mice returned the PWT in the cuff to pre-surgery levels. This is reminiscent of data obtained in the rat after systemic (i.p.) injection of another AChE antagonist, donepezil, in the spared nerve injury model (Kimura, Hayashida, Eisenach, Saito, \& Obata, 2013) demonstrating that 0.6 and $1 \mathrm{mg} / \mathrm{kg}$ of i.p. donepezil were antihyperalgesic in a mechanical paw withdrawal test, while they showed no effect before the ligature. Concerning the thermal sensory modality, i.t. injections of AChE inhibitors are also analgesic in naïve rats (Chiari, et al., 1999; Naguib \& Yaksh, 1994), and a study performed in mice showed again the anti-hyperalgesic effect of very low doses of i.t. neostigmine (another AChE inhibitor, 3ng) in the tail flick test in the SNL model, while higher doses (from 115 ng i.t.) were required for analgesia in control mice (Takasu, et al., 2006). Interestingly, nicotinic agonists also have analgesic properties after i.t. injection in the PNL mouse model, both in mechanical and thermal tests, at doses $(10 \mathrm{nmol}$ nicotine, $0.3 \mathrm{nmol}$ epibatidine) inefficient in sham mice (Rashid \& Ueda, 2002). This, together with our results, strongly suggests that major changes occur at the receptor level, either in density or in composition.

Alterations in the expression of nAChRs have been reported in neuropathic conditions. The expression of the $\alpha 6$ nAChR subunit in murine DRGs was demonstrated to correlate with the mechanical allodynia produced after neuropathy (Wieskopf, et al., 2015), while the mRNA level of the $\alpha 5$ and $\beta 2$ nAChR subunits is increased in neuropathic rats (Yang, et al., 2004). A specific study of alterations of 
nAChRs on the targets of cholinergic neurons in the dorsal horn of the spinal cord should help elucidate the changes occurring in the mouse cuff model and possibly explaining the plasticity of the cholinergic tone. In this study, we have focused on the spinal nicotinic tone because we have revealed the cholinergic tone through antagonization of nAChRs by mecamylamine. Mecamylamine is a general nicotinic antagonist (Papke, Sanberg, \& Shytle, 2001), but i.t. hexamethonium or the $\alpha 4 \beta 2$ nAChR antagonist DH $\beta E$, or the $\alpha 3 \beta 2 * / \alpha 6 \beta 2 *$ nAChR antagonist, $\alpha$-CTX-MII, have been shown to

358 have a similar effect in mice or rats (Rashid, et al., 2006; Rashid \& Ueda, 2002; Yalcin, Charlet, et al., 359 2011; Young, Wittenauer, McIntosh, \& Vincler, 2008). In addition, the allodynic effect of nicotinic antagonists is lost in nAChR $\beta 2$ knock-out mice (this study and (Yalcin, Charlet, et al., 2011)), further confirming the involvement of $\beta 2$-containing nAChRs. In contrast, the $\alpha 7 \mathrm{nAChR}$ antagonist MLA (10 nmol) has no effect, suggesting that $\alpha 7 \mathrm{nAChRs}$ are not involved in the cholinergic tone in mice (Rashid, et al., 2006; Rashid \& Ueda, 2002; Yalcin, Charlet, et al., 2011). However, it should be noted that the increased ACh level induced by AChE inhibition could act both on nicotinic and muscarinic post-synaptic receptors. In naïve rats, the analgesic effect of neostigmine on thermal responses has been shown to be mediated by muscarinic receptors in males but muscarinic and nicotinic receptors in females (Chiari, et al., 1999; P. M. Lavand'homme \& Eisenach, 1999). However muscarinic antagonists (atropine, pirenzepine, 4-DAMP, or methoctramine) had no effect on von Frey responses when injected i.t. in naïve mice (Honda, et al., 2002 ; Paqueron, Conklin, \& Eisenach, 2003), suggesting that there might be species or sensory modality differences.

371 In this study, we have demonstrated that the spinal cholinergic tone modulating nociceptive responses

372 was still present, and even more efficient, after peripheral nerve injury. Our data do not support a

373 change in the number or electrophysiological properties of dorsal horn cholinergic interneurons, but 374 rather favor a modification occurring at the post-synaptic site. Overall, the increased analgesic effect of i.t. AChE antagonists in neuropathic conditions is promising. Until now, AChE inhibitors have mainly been used in the clinics in situations of acute pain (parturition or post-operative (Eisenach, 2009)). The fact that lower doses (thus with fewer side effects) could be efficient in chronic pain conditions opens new avenues for the treatment of neuropathic pain. 
This study utilized wild-type and transgenic CD1 and C57BL/6 mice between 3 - 10 weeks. Two different transgenic lines have been used: ChAT::EGFP CD1 (von Engelhardt, Eliava, Meyer, Rozov, \& Monyer, 2007) and $\beta 2 *-n A C h R$ knock-out (KO) C57BL/6J mice (Picciotto, et al., 1995). The animals were group-housed between two to six animals per cage and maintained on a 12-hour light/dark cycles with food and water provided ad libitum. The animal facility Chronobiotron All protocols were approved by the "Comité d'Ethique en Matière d'Expérimentation Animale de Strasbourg” (CREMEAS, CEEA35) and the Ministère de l'enseignement supérieur, de la recherche et de l'innovation under the reference 02729.01.

\subsection{Cuff surgery and behavioral assessment}

The cuff model is used to induce neuropathic pain in mice (Benbouzid, et al., 2008). The surgery was carried out under ketamine (1 $\mu \mathrm{l} / \mathrm{g}$ - Imalgene1000, Merial), azepromazin (0,60 $\mu \mathrm{l} / \mathrm{g}$ - Calmivet, Vetoquinol) and medetomidine (1,18 $\mu \mathrm{l} / \mathrm{g}$ - Domitor, Orion pharma/Elanco) anesthesia. The surgery was performed as previously described (Yalcin, et al., 2014). Briefly, a $2 \mathrm{~mm}$ section of split PE-20 polyethylene tubing (Harvard apparatus, Les Ulis, France) was placed around the right common branch of sciatic nerve in the cuff mice group. Sham-operated mice underwent the same surgical procedure without cuff implantation. After performing the surgery, Atipamezol chlorhydrate (Antisedan, Orion pharma/Vetoquinol) mix (10 $\mu \mathrm{l} / \mathrm{g}$ of animal), an antidote to medetomidine, was provided to speed up the recovery from the anesthesia. After a one-week recovery period, the neuropathic phenotype was assessed with von Frey tests.

Intrathecal (i.t.) injections were performed under gaseous anesthesia (3\% Isoflurane for induction and 2\% for maintenance) as previously described (Yalcin, Charlet, et al., 2011). We assessed the role of spinal cholinergic modulation on mechanical transmission in naïve and neuropathic CD1 mice with von Frey filaments (Yalcin, et al., 2010). Mice were habituated for 15 minutes in clear Plexiglas boxes

406 (7cm $\times 9 \mathrm{~cm} \times 7 \mathrm{~cm})$ on an elevated mesh screen. Filaments of increasing diameter were slowly 407 brought to the plantar surface of each hind paw and removed after a slight bent. The mechanical threshold is determined as the first filament inducing at least three withdrawals out of five consecutive trials. The results were expressed in grams. For naïve animals, the left and right paw data were averaged for each time point. The mice were tested prior to injection or surgery to establish the

411 baselines. A minimum of three tests were performed over 3 to 7 days to determine the mechanical withdrawal threshold. Motor coordination was tested using a rotarod (Bioseb). Mice were trained on 2 
414 falling for at least 60 secs. On the experimental day, the rod was programmed to accelerate from 0 to

$41540 \mathrm{rpm}$ over $300 \mathrm{~s}$. Each mouse was allowed three trials where the average rpm and time at the point

416 of failure was recorded to establish the baseline. Subsequently, mecamylamine was intrathecally

417 injected and the mice were tested again 3 times starting after 15 min post-injection.

From 7 to 24 days post-surgery, ChAT::EGFP CD1 mice were anesthetized with a mix of i.p. ketamine (200mg/kg - Imalgene 1000, Merial) and xylazine (20 mg/kg - Rompun 2\%, Bayer). The animals were transcardially perfused for approximately 3 minutes with ice-cold sucrose-based Artificial Cerebral Spinal Fluid (ACSF) composed of (in mM): 252 Sucrose, $2.5 \mathrm{KCl}, 2 \mathrm{CaCl} 2,2$ MgCl2, 10 Glucose, 26 NaHCO3, 1.25 NaH2PO4, 2 kynurenic acid, continuously bubbled with carbogen $\left(95 \% \mathrm{O}_{2} / 5 \% \mathrm{CO}_{2}\right)$. Two approaches were used to extract the spinal cord: hydraulic extrusion (Chery \& De Koninck, 1999) or laminectomy (Flynn, Brichta, Galea, Callister, \& Graham, 2011). A 200 to $300 \mu \mathrm{m}$ horizontal slice (HS) was performed, containing the first four laminae of the DH. We also performed 350 to $400 \mu \mathrm{m}$ thick transverse slices (TS). The slices were allowed a onehour recovery period prior to recording at room temperature in oxygenated ACSF containing (in $\mathrm{mM}$ ): $126 \mathrm{NaCl}, 2.5 \mathrm{KCl}, 2 \mathrm{CaCl}$, 2 MgCl2, 10 Glucose, 26 NaHCO3, 1.25 NaH2PO4. produced under the control of the cholineacetyltransferase (ChAT) promoter. Cells were recorded in the lumbar cord, in the area corresponding to termination of the sciatic nerve (end of L3 - L6 spinal segments). In HS, LIII/IV was identified by few ChAT::EGFP neurons located on the surface of the first slice (positioned upside-down). In TS, LIII/IV was identified as the laminae just ventral to the translucent substantia gelatinosa (LII), or ventral to the EGFP+ cholinergic plexus (Mesnage, et al., 2011). For voltage clamp experiments, a cesium (Cs) based intracellular solution was used (in $\mathrm{mM}$ ): 80 Cs2SO4, $5 \mathrm{KCl}, 2 \mathrm{MgCl} 2,10$ HEPES, 10 Biocytin ( $\mathrm{pH}=7.3$ ). The cells were maintained at -60 $\mathrm{mV}$ and $0 \mathrm{mV}$ for excitatory and inhibitory post-synaptic currents respectively. For current clamp recordings, a K-based intracellular solution was used (in mM): 136 CH3KO3S, 2 MgCl2, 10 HEPES, 10 Biocytin. For both intracellular solutions, the liquid junction potential was not corrected. The recordings were made through the Axopatch 200A (Axon instruments) or the Multiclamp 700A (Molecular devices). The current traces were filtered at $5 \mathrm{kHz}$ before digitalization via a digitizer (BNC-2110, National Instruments) at $20 \mathrm{kHz}$. Data acquisition was performed using WinEDR and

444 WinWCP softwares (Strathclyde Electrophysiology Software, John Dempster, University of 445 Strathclyde, Glasgow, UK).

446 For voltage clamp, the synaptic events (recorded over a 5-minute period) were detected with the 447 threshold search method with WinEDR software. The threshold was set, for miniature and 
spontaneous EPSCs, to absolute amplitudes $\geq 2 \mathrm{pA}$ and a duration $\geq 1.25 \mathrm{~ms}$. For miniature and spontaneous IPSCs, the thresholds were set to absolute amplitudes $\geq 3 \mathrm{pA}$ and a duration $\geq 1.75 \mathrm{~ms}$. A baseline track-time of $5 \mathrm{~ms}$ was implemented prior to each event. Following the automatic detection by the software, all captured events were inspected individually, and only events that possessed a quick rise followed by an exponential decay were kept. The absolute frequency is calculated as the number of events over the total duration of the recording. The peak amplitudes were measured for accepted events via semi-automated procedures in WinWCP. However, individual events were excluded if they contained overlapping events or had an unstable baseline. The frequency and amplitude of currents were analyzed over a 5-minute duration. For current clamp, the firing patterns were classified as previously reported (Prescott \& De Koninck, 2002).

\subsection{In vivo electrophysiological recordings}

Adult CD1 males were anesthetized with urethane ( $2.5 \mathrm{~g} / \mathrm{kg}$, i.p.) and the trachea was cannulated. Rectal temperature was continuously monitored, and the animal was maintained at $35.5^{\circ} \mathrm{C}$ using a heating pad (TC-1000; Bioseb). The mouse was placed in a stereotaxic and spinal frame with 2 clamps fixed on its vertebra to immobilize the vertebral column. A laminectomy was performed to expose L3L5 segments of the spinal cord, and a small chamber (approximately $0.1 \mathrm{ml}$ ) was created with $2 \%$ agar around the exposed lumbar spinal cord. After removal of the dura, the spinal cord was covered with saline ( $\mathrm{NaCl} 0.9 \%)$ or with drugs diluted in saline (see below).

Single-unit extracellular recordings from spinal dorsal horn neurons were performed as previously described (Medrano, et al., 2016). Only neurons responding to mechanical stimulation of the ipsilateral hind paw were included in this study. Recordings were made with a glass electrode (Harvard Apparatus, Holliston, MA) filled with 0.5M CH3COOK (resistance: 15-25 mV). A motorized micromanipulator (Narishige, Tokyo, Japan) was used to gradually descend the electrode with 4-mm steps until the single-unit activity of a neuron was recorded. The recording electrode was inserted at depths $\leq 500 \mathrm{~mm}$ from the surface of the spinal cord (corresponding to lamina I to $\mathrm{V}$ ). The signal was amplified (IR-183; Cygnus Technology, Delaware Water Gap, PA), filtered at 0.3 to $3 \mathrm{kHz}$ (Brownlee amplifier; AutoMate Scientific, Berkeley, CA), and digitized at $20 \mathrm{kHz}$ with a MICRO31401 (CED, Cambridge, United Kingdom). Data were analyzed offline with Spike 2 software (CED). The cutaneous receptive field of the recorded neuron was identified by touching the ipsilateral hind paw. The response to a non-nociceptive mechanical stimulus, touch, and to a nociceptive one, pinch, was determined as described before (Medrano, et al., 2016)). The touch stimulus was applied by brushing the skin with a camel's hair brush for 10 seconds (8-10 times). The pinch stimulus was applied by means of small serrated forceps (Graefe forceps; Fine Scientific Tools, Vancouver, Canada) for 10 seconds. 

applied directly at the surface of the dorsal horn using the agar chamber described above. Stock solutions were first prepared in water at $100 \mathrm{x}$ their final concentration, stored at $-20{ }^{\circ} \mathrm{C}$ and, on the day of the experiment, diluted in saline to their final concentration. The drug was applied topically onto the recording site of the spinal cord after careful removal of saline of the pool. Ten minutes following drug application, the response of neurons to the mechanical stimuli was tested (Medrano, et al., 2016). All solutions were applied at $34-35^{\circ} \mathrm{C}$.

\subsection{Tissue fixing and staining}

The animals were transcardially perfused under pentobarbital (Ceva Sante Animal, 54.7mg/ml, i.p. injection) with $0.1 \mathrm{M}$ phosphate buffer (PB, $\mathrm{pH}$ 7.4) followed by $4 \%$ Paraformaldehyde (PFA) in PB for 15 minutes. The lumbar part of the spinal cord was sliced in $40 \mu \mathrm{m}$-thick transverse sections using a vibrating blade microtome (VT 1000S, Leica, Rueil-Malmaison, France), and serially collected in wells. The sections were washed three times with PBS and saturated with PBS/ 0.5\% Triton X-100/ $5 \%$ donkey serum at room temperature for 45 minutes. The sections were incubated at room temperature overnight in PBS/ $0.5 \%$ Triton X-100/ 1\% donkey serum with a goat polyclonal ChAT antibody (1:500 dilution, Chemicon Millipore, AB 144P). After three PBS washes, the slides were incubated with a CY3 anti-goat antibody (1:400 dilution, Jackson Immunoresearch) for 2 hr. The sections were finally washed three times with PBS and mounted with fluorescent mounting media (Dako, Les Ulis, France). Slides were examined under fluorescence using a microscope (Leica) and an exhaustive counting of fluorescent neurons was made in both the contralateral and ipsilateral DH in all sections between L4 and L6.

\subsection{Statistics}

504 The statistical analysis was performed with the statistical software R (version 3.4.1) in conjunction 505 with Graphpad software (Prism 7 for Mac, GraphPad Software, Inc., San Diego, CA, USA). For in 506 vitro data, almost all statistical comparisons for frequency, amplitude and cell properties, considering 507 the various dependent variables, were made with the ANOVA function. The original or transformed 508 (log or inverse function) datasets were verified to show a normal distribution (Shapiro-Wilk normality 509 test). For post-hoc comparisons, interactions with only one or two variables were made with the 510 Bonferroni test with multi-comparison correction. A more complex 3x3x3 contingency table was 511 performed online at http://vassarstats.net (Log-Linear Analysis for an AxBxC Contingency Table 512 found under Frequency data tab) for firing pattern, rebound spike observations and rheobase. For in 513 vivo and in vitro data passing requirements for parametric tests, statistical evaluation was carried out 514 with repeated measures 2-way ANOVA. In addition, the depths for in vivo recordings were compared 515 with unpaired t-test. 
516 As the vonFrey data is a discrete ordinal data, it did not pass the conditions to use parametric tests. We

517 thus analyzed it using the non-parametric test "nparLD”, provided as a plug-in by the statistical

518 software R (Noguchi, Gel, Brunner, \& Konietschke, 2012). nparLD provides an ANOVA type

519 multiple-factor analysis taking into account a longitudinal variable (time) along with multiple

520 dependent (right vs left paw) and independent (surgery; drug treatment) variables. The Bonferroni test

521 was also used for post-hoc multi-comparisons against saline.

522 The significance level was set at $\mathrm{P}<0.05$ and data were expressed as mean \pm SEM for graphs.

\section{Acknowledgements:}

524 We gratefully acknowledge the support from the University of Strasbourg Institute for Advanced 525 Study (USIAS) and ANR-13-JSV4-0003-01 GRANT to MCE. DD is the recipient of a Region Alsace 526 doctoral fellowship, YML of a Fondation pour la Recherche Médicale post-doctoral fellowship, and 527 SK was supported by the NeuroTime Erasmus Mundus Joint Doctorate Neuroscience PhD program 528 (funded by the European Commission), and CB a recipient of a fellowship from the French Ministry 529 of Higher Education and Research. The authors express their gratitude towards J.L. Rodeau for his 530 expert assistance on the statistical approaches, Philippe Isope and Yves De Koninck for critical 531 readings; and Sophie Reibel-Foisset and the Chronobiotron (UMS 3415, Centre National de la 532 Recherche Scientifique) for mice handling. The authors have no conflicts of interest to declare. 


\section{References:}

Abraira, V. E., Kuehn, E. D., Chirila, A. M., Springel, M. W., Toliver, A. A., Zimmerman, A. L., et al. (2017). The Cellular and Synaptic Architecture of the Mechanosensory Dorsal Horn. Cell, 168(1-2), 295-310 e219, S0092-8674(16)31684-1 [pii]

10.1016/j.cell.2016.12.010.

Alba-Delgado, C., El Khoueiry, C., Peirs, C., Dallel, R., Artola, A., \& Antri, M. (2015). Subpopulations of PKCgamma interneurons within the medullary dorsal horn revealed by electrophysiologic and morphologic approach. Pain, 156(9), 1714-1728, 10.1097/j.pain.0000000000000221.

Barber, R. P., Phelps, P. E., Houser, C. R., Crawford, G. D., Salvaterra, P. M., \& Vaughn, J. E. (1984). The morphology and distribution of neurons containing choline acetyltransferase in the adult rat spinal cord: an immunocytochemical study. Journal of Comparative Neurology, 229(3), 329-346.

Benbouzid, M., Pallage, V., Rajalu, M., Waltisperger, E., Doridot, S., Poisbeau, P., et al. (2008). Sciatic nerve cuffing in mice: a model of sustained neuropathic pain. Eur J Pain, 12(5), 591-599, S1090-3801(07)00654-4 [pii]

10.1016/j.ejpain.2007.10.002.

Breivik, H., Collett, B., Ventafridda, V., Cohen, R., \& Gallacher, D. (2006). Survey of chronic pain in Europe: prevalence, impact on daily life, and treatment. Eur J Pain, 10(4), 287-333, S10903801(05)00086-8 [pii]

10.1016/j.ejpain.2005.06.009.

Chen, S. R., \& Pan, H. L. (2003). Spinal GABAB receptors mediate antinociceptive actions of cholinergic agents in normal and diabetic rats. Brain Res, 965(1-2), 67-74, S0006899302041239 [pii].

Chen, S. R., \& Pan, H. L. (2004). Activation of muscarinic receptors inhibits spinal dorsal horn projection neurons: role of GABAB receptors. Neuroscience, 125(1), 141-148, 10.1016/j.neuroscience.2004.01.015

S0306452204000521 [pii].

Cheng, L., Duan, B., Huang, T., Zhang, Y., Chen, Y., Britz, O., et al. (2017). Identification of spinal circuits involved in touch-evoked dynamic mechanical pain. Nat Neurosci, 20(6), 804-814, nn.4549 [pii]

$10.1038 / \mathrm{nn} .4549$.

Chery, N., \& De Koninck, Y. (1999). Junctional versus extrajunctional glycine and GABA(A) receptormediated IPSCs in identified lamina I neurons of the adult rat spinal cord. J Neurosci, 19(17), $7342-7355$.

Chiari, A., Tobin, J. R., Pan, H. L., Hood, D. D., \& Eisenach, J. C. (1999). Sex differences in cholinergic analgesia I: a supplemental nicotinic mechanism in normal females. Anesthesiology, 91(5), 1447-1454.

Cordero-Erausquin, M., Inquimbert, P., Schlichter, R., \& Hugel, S. (2016). Neuronal networks and nociceptive processing in the dorsal horn of the spinal cord. Neuroscience, S03064522(0316)30421-30423, S0306-4522(16)30421-3 [pii]

10.1016/j.neuroscience.2016.08.048.

Eisenach, J. C. (2009). Epidural neostigmine: will it replace lipid soluble opioids for postoperative and labor analgesia? Anesth Analg, 109(2), 293-295, 109/2/293 [pii]

10.1213/ane.0b013e3181a891c2.

Eisenach, J. C., Detweiler, D. J., Tong, C., D'Angelo, R., \& Hood, D. D. (1996). Cerebrospinal fluid norepinephrine and acetylcholine concentrations during acute pain. Anesth Analg, 82(3), 621-626. 
Flynn, J. R., Brichta, A. M., Galea, M. P., Callister, R. J., \& Graham, B. A. (2011). A horizontal slice preparation for examining the functional connectivity of dorsal column fibres in mouse spinal cord. J Neurosci Methods, 200(2), 113-120, S0165-0270(11)00352-9 [pii]

10.1016/j.jneumeth.2011.06.017.

Foster, E., Wildner, H., Tudeau, L., Haueter, S., Ralvenius, W. T., Jegen, M., et al. (2015). Targeted ablation, silencing, and activation establish glycinergic dorsal horn neurons as key components of a spinal gate for pain and itch. Neuron, 85(6), 1289-1304, S08966273(15)00143-9 [pii]

10.1016/j.neuron.2015.02.028.

Gassner, M., Leitner, J., Gruber-Schoffnegger, D., Forsthuber, L., \& Sandkuhler, J. (2013). Properties of spinal lamina III GABAergic neurons in naive and in neuropathic mice. Eur J Pain, 17(8), 1168-1179, 10.1002/j.1532-2149.2013.00294.x.

Hama, A., \& Menzaghi, F. (2001). Antagonist of nicotinic acetylcholine receptors (nAChR) enhances formalin-induced nociception in rats: tonic role of $n A C h R s$ in the control of pain following injury. Brain Res., 888(1), 102-106.

Hartvig, P., Gillberg, P. G., Gordh, T., Jr., \& Post, C. (1989). Cholinergic mechanisms in pain and analgesia. Trends Pharmacol.Sci., Suppl, 75-79.

Honda, K., Koga, K., Moriyama, T., Koguchi, M., Takano, Y., \& Kamiya, H. O. (2002). Intrathecal alpha2 adrenoceptor agonist clonidine inhibits mechanical transmission in mouse spinal cord via activation of muscarinic M1 receptors. Neurosci.Lett., 322(3), 161-164.

Hwang, J. H., Hwang, K. S., Leem, J. K., Park, P. H., Han, S. M., \& Lee, D. M. (1999). The antiallodynic effects of intrathecal cholinesterase inhibitors in a rat model of neuropathic pain. Anesthesiology, 90(2), 492-499.

Imlach, W. L., Bhola, R. F., Mohammadi, S. A., \& Christie, M. J. (2016). Glycinergic dysfunction in a subpopulation of dorsal horn interneurons in a rat model of neuropathic pain. Sci Rep, 6 , 37104, srep37104 [pii]

$10.1038 /$ srep37104.

Inquimbert, P., Moll, M., Latremoliere, A., Tong, C. K., Whang, J., Sheehan, G. F., et al. NMDA Receptor Activation Underlies the Loss of Spinal Dorsal Horn Neurons and the Transition to Persistent Pain after Peripheral Nerve Injury. Cell Rep, 23(9), 2678-2689, S2211-

1247(18)30705-8 [pii]

10.1016/j.celrep.2018.04.107.

Iura, A., Takahashi, A., Hakata, S., Mashimo, T., \& Fujino, Y. (2016). Reductions in tonic GABAergic current in substantia gelatinosa neurons and GABAA receptor delta subunit expression after chronic constriction injury of the sciatic nerve in mice. Eur J Pain, 20(10), 1678-1688, 10.1002/ejp.891.

Kimura, M., Hayashida, K., Eisenach, J. C., Saito, S., \& Obata, H. (2013). Relief of hypersensitivity after nerve injury from systemic donepezil involves spinal cholinergic and gamma-aminobutyric acid mechanisms. Anesthesiology, 118(1), 173-180, 10.1097/ALN.0b013e318277a81c.

Lavand'homme, P., Pan, H. L., \& Eisenach, J. C. (1998). Intrathecal neostigmine, but not sympathectomy, relieves mechanical allodynia in a rat model of neuropathic pain. Anesthesiology, 89(2), 493-499.

Lavand'homme, P. M., \& Eisenach, J. C. (1999). Sex differences in cholinergic analgesia II: differing mechanisms in two models of allodynia. Anesthesiology, 91(5), 1455-1461.

Leitner, J., Westerholz, S., Heinke, B., Forsthuber, L., Wunderbaldinger, G., Jager, T., et al. (2013). Impaired excitatory drive to spinal GABAergic neurons of neuropathic mice. PLoS One, 8(8), e73370, 10.1371/journal.pone.0073370

PONE-D-12-18620 [pii].

Lu, Y., Dong, H., Gao, Y., Gong, Y., Ren, Y., Gu, N., et al. (2013). A feed-forward spinal cord glycinergic neural circuit gates mechanical allodynia. J Clin Invest, 123(9), 4050-4062, 70026 [pii]

10.1172/JCl70026. 
McDonough, J. H., Jr., \& Shih, T. M. (1995). A study of the N-methyl-D-aspartate antagonistic properties of anticholinergic drugs. Pharmacol Biochem Behav, 51(2-3), 249-253, 10.1016/0091-3057(94)00372-p.

Medrano, M. C., Dhanasobhon, D., Yalcin, I., Schlichter, R., \& Cordero-Erausquin, M. (2016). Loss of inhibitory tone on spinal cord dorsal horn spontaneously and nonspontaneously active neurons in a mouse model of neuropathic pain. Pain, 157(7), 1432-1442, 10.1097/j.pain.0000000000000538.

Mesnage, B., Gaillard, S., Godin, A., Rodeau, J. L., Hammer, M., von Engelhardt, J., et al. (2011). Morphological and functional characterization of cholinergic interneurons of the dorsal horn of the mouse spinal cord. J Comp Neurol, 519(16), 3139-3158.

Miles, G. B., Hartley, R., Todd, A. J., \& Brownstone, R. M. (2007). Spinal cholinergic interneurons regulate the excitability of motoneurons during locomotion. Proc Natl Acad Sci U S A, 104(7), 2448-2453, 0611134104 [pii]

\subsection{3/pnas.0611134104.}

Miranda, H. F., Sierralta, F., \& Pinardi, G. (2002). Neostigmine interactions with non steroidal antiinflammatory drugs. Br J Pharmacol, 135(7), 1591-1597, 10.1038/sj.bjp.0704599.

Moore, K. A., Kohno, T., Karchewski, L. A., Scholz, J., Baba, H., \& Woolf, C. J. (2002). Partial peripheral nerve injury promotes a selective loss of GABAergic inhibition in the superficial dorsal horn of the spinal cord. J Neurosci, 22(15), 6724-6731, 20026611

22/15/6724 [pii].

Mosconi, T., \& Kruger, L. (1996). Fixed-diameter polyethylene cuffs applied to the rat sciatic nerve induce a painful neuropathy: ultrastructural morphometric analysis of axonal alterations. Pain, 64(1), 37-57, 10.1016/0304-3959(95)00077-1.

Naguib, M., \& Yaksh, T. L. (1994). Antinociceptive effects of spinal cholinesterase inhibition and isobolographic analysis of the interaction with mu and alpha 2 receptor systems. Anesthesiology, 80(6), 1338-1348.

Noguchi, K., Gel, Y. R., Brunner, E., \& Konietschke, F. (2012). nparLD: An R Software Package for the Nonparametric Analysis of Longitudinal Data in Factorial Experiments. Journal of Statistical Software, 50(12), 23, 10.18637/jss.v050.i12.

O'Dell, T. J., \& Christensen, B. N. (1988). Mecamylamine is a selective non-competitive antagonist of $\mathrm{N}$-methyl-D-aspartate- and aspartate-induced currents in horizontal cells dissociated from the catfish retina. Neurosci Lett, 94(1-2), 93-98, 10.1016/0304-3940(88)90276-5.

Olave, M. J., Puri, N., Kerr, R., \& Maxwell, D. J. (2002). Myelinated and unmyelinated primary afferent axons form contacts with cholinergic interneurons in the spinal dorsal horn. Exp.Brain Res., 145(4), 448-456.

Papke, R. L., Sanberg, P. R., \& Shytle, R. D. (2001). Analysis of mecamylamine stereoisomers on human nicotinic receptor subtypes. J Pharmacol Exp Ther, 297(2), 646-656.

Paqueron, X., Conklin, D., \& Eisenach, J. C. (2003). Plasticity in action of intrathecal clonidine to mechanical but not thermal nociception after peripheral nerve injury. Anesthesiology, 99(1), 199-204.

Pawlowski, S. A., Gaillard, S., Ghorayeb, I., Ribeiro-da-Silva, A., Schlichter, R., \& Cordero-Erausquin, M. (2013). A novel population of cholinergic neurons in the macaque spinal dorsal horn of potential clinical relevance for pain therapy. J Neurosci, 33(9), 3727-3737, 33/9/3727 [pii] 10.1523/JNEUROSCI.3954-12.2013.

Peirs, C., Williams, S. P., Zhao, X., Walsh, C. E., Gedeon, J. Y., Cagle, N. E., et al. (2015). Dorsal Horn Circuits for Persistent Mechanical Pain. Neuron, 87(4), 797-812, S0896-6273(15)00671-6 [pii] 10.1016/j.neuron.2015.07.029.

Petitjean, H., Pawlowski, S. A., Fraine, S. L., Sharif, B., Hamad, D., Fatima, T., et al. (2015). Dorsal Horn Parvalbumin Neurons Are Gate-Keepers of Touch-Evoked Pain after Nerve Injury. Cell Rep, 13(6), 1246-1257, 10.1016/j.celrep.2015.09.080. 
Picciotto, M., Zoli, M., L,na, C., Bessis, A., Lallemand, Y., Lenovere, N., et al. (1995). Abnormal avoidance learning in mice lacking functional high-affinity nicotine receptor in the brain. Nature, 374, 65-67.

Polgar, E., Gray, S., Riddell, J. S., \& Todd, A. J. (2004). Lack of evidence for significant neuronal loss in laminae I-III of the spinal dorsal horn of the rat in the chronic constriction injury model. Pain, 111(1-2), 144-150, 10.1016/j.pain.2004.06.011

S0304395904002994 [pii].

Prescott, S. A., \& De Koninck, Y. (2002). Four cell types with distinctive membrane properties and morphologies in lamina I of the spinal dorsal horn of the adult rat. J Physiol, 539, 817-836.

Rashid, M. H., Furue, H., Yoshimura, M., \& Ueda, H. (2006). Tonic inhibitory role of alpha4beta2 subtype of nicotinic acetylcholine receptors on nociceptive transmission in the spinal cord in mice. Pain, 125(1-2), 125-135.

Rashid, M. H., \& Ueda, H. (2002). Neuropathy-specific analgesic action of intrathecal nicotinic agonists and its spinal GABA-mediated mechanism. Brain Res, 953(1-2), 53-62.

Ribeiro-da-Silva, A. (2004). Substantia Gelatinosa of the Spinal Cord. In G. Paxinos (Ed.), The Rat Nervous System (Vol. Third, pp. 129-148). San Diego: Elsevier Academic Press.

Santos, S. F., Rebelo, S., Derkach, V. A., \& Safronov, B. V. (2007). Excitatory interneurons dominate sensory processing in the spinal substantia gelatinosa of rat. J.Physiol, 581(Pt 1), 241-254.

Schoffnegger, D., Heinke, B., Sommer, C., \& Sandkuhler, J. (2006). Physiological properties of spinal lamina II GABAergic neurons in mice following peripheral nerve injury. J Physiol, 577(Pt 3), 869-878, jphysiol.2006.118034 [pii]

10.1113/jphysiol.2006.118034.

Scholz, J., Broom, D. C., Youn, D. H., Mills, C. D., Kohno, T., Suter, M. R., et al. (2005). Blocking caspase activity prevents transsynaptic neuronal apoptosis and the loss of inhibition in lamina II of the dorsal horn after peripheral nerve injury. J Neurosci, 25(32), 7317-7323, 25/32/7317 [pii] 10.1523/JNEUROSCI.1526-05.2005.

Takasu, K., Honda, M., Ono, H., \& Tanabe, M. (2006). Spinal alpha(2)-adrenergic and muscarinic receptors and the NO release cascade mediate supraspinally produced effectiveness of gabapentin at decreasing mechanical hypersensitivity in mice after partial nerve injury. Br.J.Pharmacol., 148(2), 233-244.

Tsuda, M., Koga, K., Chen, T., \& Zhuo, M. (2017). Neuronal and microglial mechanisms for neuropathic pain in the spinal dorsal horn and anterior cingulate cortex. J Neurochem, 141(4), 486-498, 10.1111/jnc.14001.

von Engelhardt, J., Eliava, M., Meyer, A. H., Rozov, A., \& Monyer, H. (2007). Functional characterization of intrinsic cholinergic interneurons in the cortex. J.Neurosci., 27(21), 56335642.

Wieskopf, J. S., Mathur, J., Limapichat, W., Post, M. R., Al-Qazzaz, M., Sorge, R. E., et al. (2015). The nicotinic alpha6 subunit gene determines variability in chronic pain sensitivity via crossinhibition of P2X2/3 receptors. Sci Transl Med, 7(287), 287ra272, 7/287/287ra72 [pii]

10.1126/scitranslmed.3009986.

Yalcin, I., Bohren, Y., Waltisperger, E., Sage-Ciocca, D., Yin, J. C., Freund-Mercier, M. J., et al. (2011). A time-dependent history of mood disorders in a murine model of neuropathic pain. Biol Psychiatry, 70(10), 946-953, S0006-3223(11)00743-8 [pii]

10.1016/j.biopsych.2011.07.017.

Yalcin, I., Charlet, A., Cordero-Erausquin, M., Tessier, L. H., Picciotto, M. R., Schlichter, R., et al. (2011). Nociceptive thresholds are controlled through spinal beta(2)-subunit-containing nicotinic acetylcholine receptors. Pain, 152(9), 2131-2137, S0304-3959(11)00355-1 [pii] 10.1016/j.pain.2011.05.022.

Yalcin, I., Megat, S., Barthas, F., Waltisperger, E., Kremer, M., Salvat, E., et al. (2014). The sciatic nerve cuffing model of neuropathic pain in mice. J Vis Exp(89), 10.3791/51608. 
Yalcin, I., Tessier, L. H., Petit-Demouliere, N., Waltisperger, E., Hein, L., Freund-Mercier, M. J., et al. (2010). Chronic treatment with agonists of beta(2)-adrenergic receptors in neuropathic pain. Exp Neurol, 221(1), 115-121, S0014-4886(09)00425-7 [pii] 10.1016/j.expneurol.2009.10.008.

Yang, L., Zhang, F. X., Huang, F., Lu, Y. J., Li, G. D., Bao, L., et al. (2004). Peripheral nerve injury induces trans-synaptic modification of channels, receptors and signal pathways in rat dorsal spinal cord. Eur J Neurosci, 19(4), 871-883, 3121 [pii] acetylcholine receptors tonically inhibit the transmission of nociceptive mechanical stimuli. Brain Res, 1229, 118-124, S0006-8993(08)01564-3 [pii] 10.1016/j.brainres.2008.06.086.

Zhuo, M., \& Gebhart, G. F. (1991). Tonic cholinergic inhibition of spinal mechanical transmission. Pain, 46(2), 211-222. 

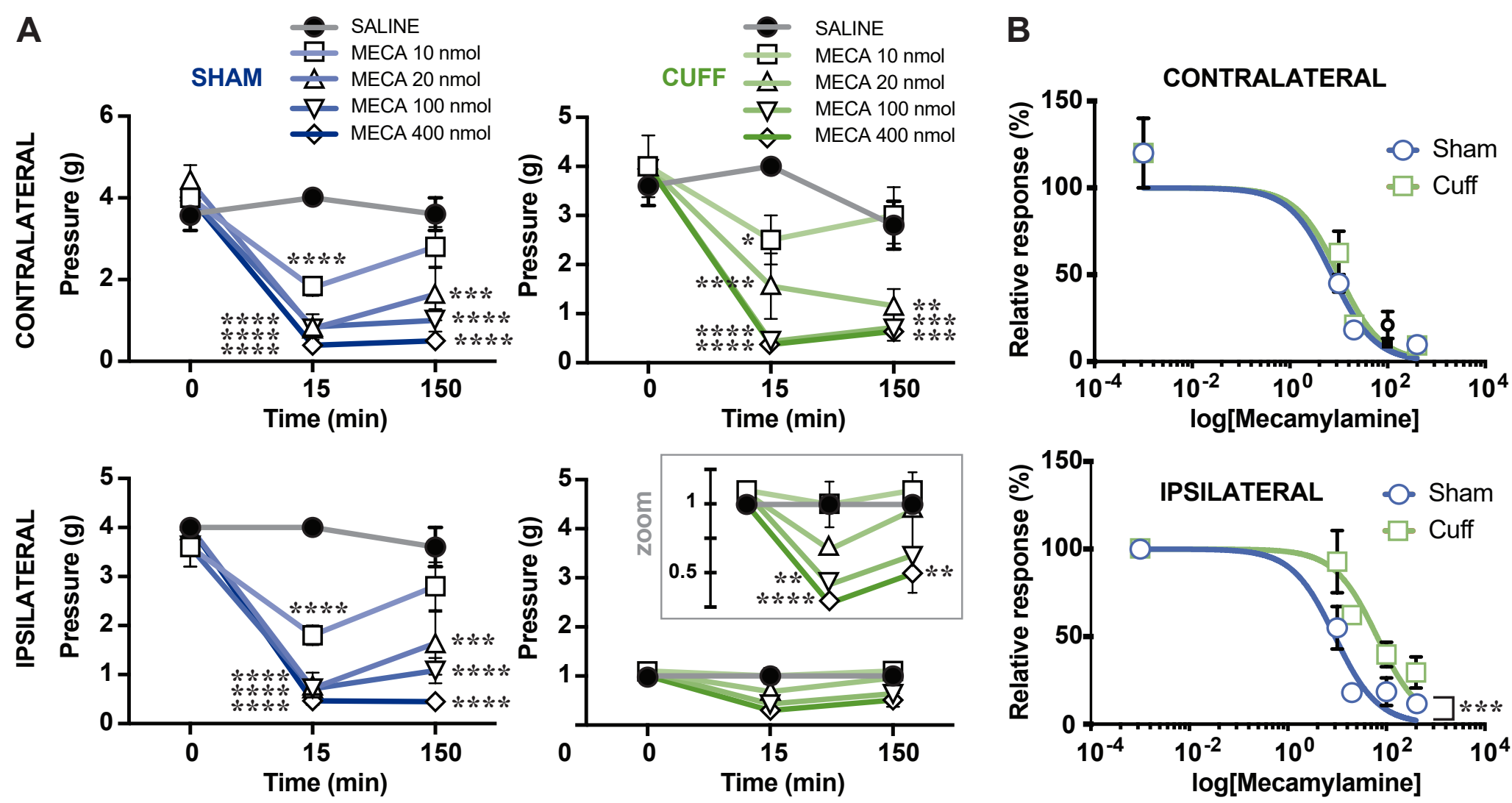

Figure 2: Mecamylamine shows that a spinal cholinergic tone is still present after neuropathy

A: Time course of the effect of intrathecal mecamylamine on mechanical threshold of sham and cuff mice (groups $\mathrm{n}=4-5$ ). For the contrateral side (Top panels), there was a significant effect of the Drug (saline, 10-400 nM mecamylamine, $\mathrm{p}=1.901 \mathrm{e}-19$, nparLD) and Time $(0,15$ and 150 min post i.t. injection, $\mathrm{p}=1.95 \mathrm{e}-67$, nparLD) but not of the Surgery ( sham vs. cuff, $\mathrm{p}=0.702$, nparLD), as well as a significant interaction between Drug and Time $(\mathrm{p}=$ $1.79 \mathrm{e}-16$, nparLD). For the ipsilateral side (Bottom panels), there was a significant effect of Surgery $(\mathrm{p}=8.64 \mathrm{e}-19)$, Drug $(\mathrm{p}=6.36 \mathrm{e}-15)$ and Time $(\mathrm{p}=3.07 \mathrm{e}-53)$, as well as a significant interaction between Drug and Time $(\mathrm{p}=$ 1.94e-16) and between Surgery, Time and Drug $(\mathrm{p}=0.0354)$. While treatment with 10 and 20 nmol mecamylamine had no effect, 100 and $400 \mathrm{nmol}$ mecamylamine further potentiated the mechanical allodynia in the ipsilateral paw. $* * * * \mathrm{p}<0.0001, * * * \mathrm{p}<0.001 * * \mathrm{p}<0.01, * \mathrm{p}<0.05$ vs. saline, Bonferroni's multiple comparisons test.

B: Dose response curve of mecamylamine on responses of sham and cuff animals. Neuropathy induced a shift to the right to the response to mecamylamine (Ipsilateral side: sham vs. cuff $\mathrm{p}<0.001$; Least square fit). 


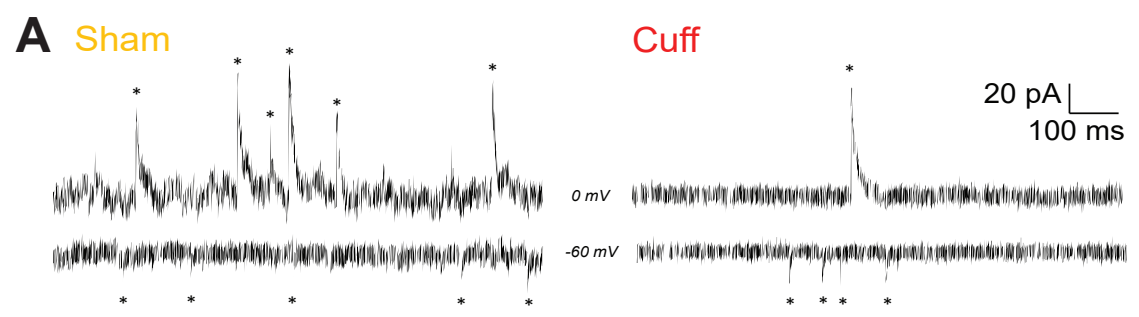

B Lamina II - Frequency
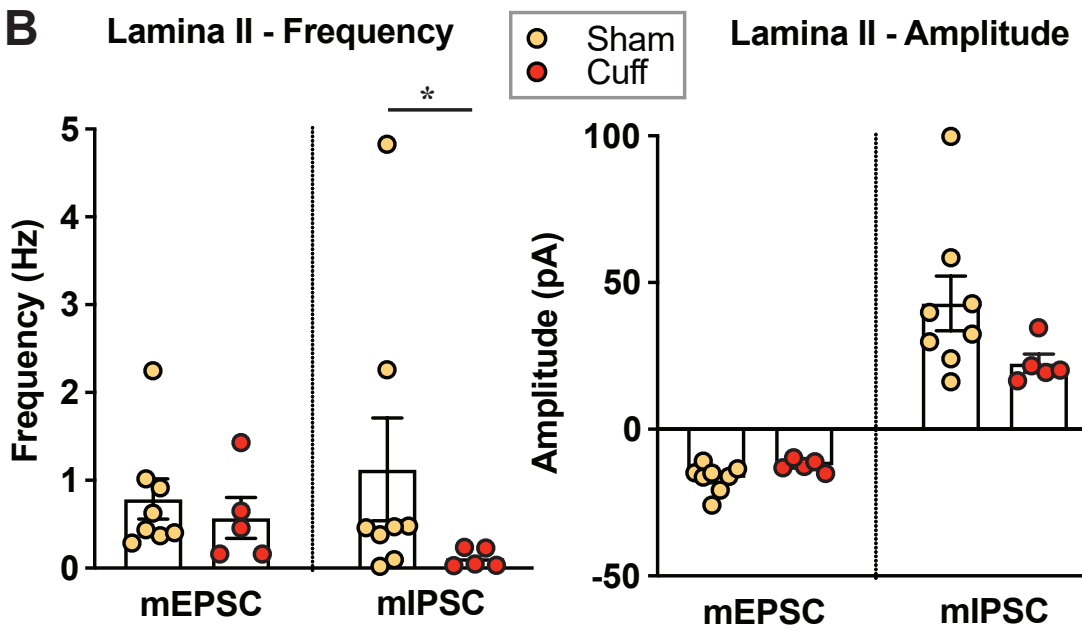

Figure 3: In vitro electrophysiological characterization of the cuff model

A: Representative patch-clamp recordings from LII neurons illustrating miniature excitatory (mEPSC, at $-60 \mathrm{mV}$ ) and inhibitory (mIPSC, at $0 \mathrm{mV}$ ) postsynaptic currents

B: (left) Frequency changes in mEPSC and mIPSC recorded LII neurons ( $n=5-9$ neurons per group): a reduction was observed in mIPSCs frequency (Right) but not mEPSCs (Left) in cuff mice (respectively, $\mathrm{p}=0.042$ and $\mathrm{p}=0.586$; Kolmogorov-Smirnov test ). (right) Analysis of the amplitude of the same currents demonstrates no effect of the surgery (mEPSC: $p=0.0793$; mIPSC: $p=0.0793$;

Kolmogorov-Smirnov test) 
$0 \mathrm{mV}$. $-60 \mathrm{mV}$

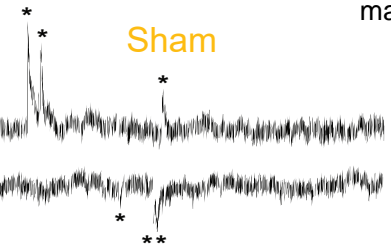
made available under aCC-BY-NC-ND 4.0 International license.

Cuff
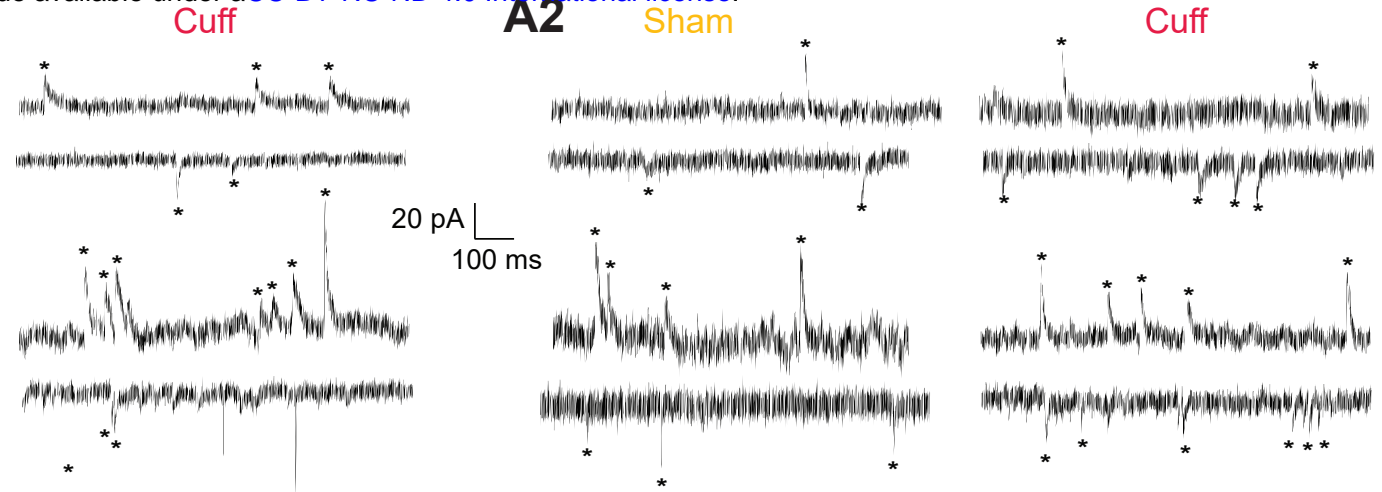

Non-EGFP

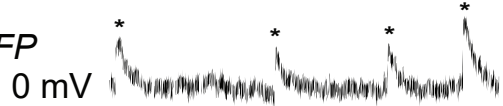
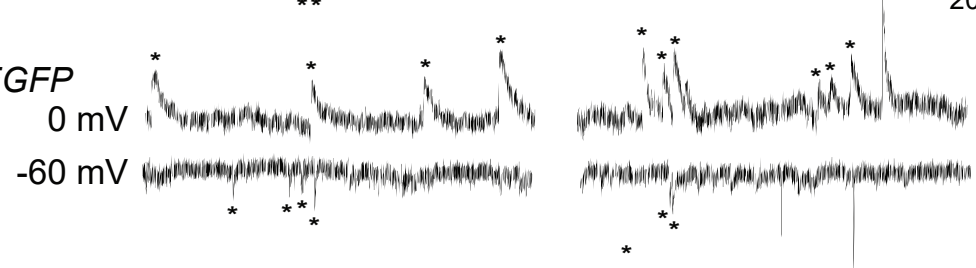

B
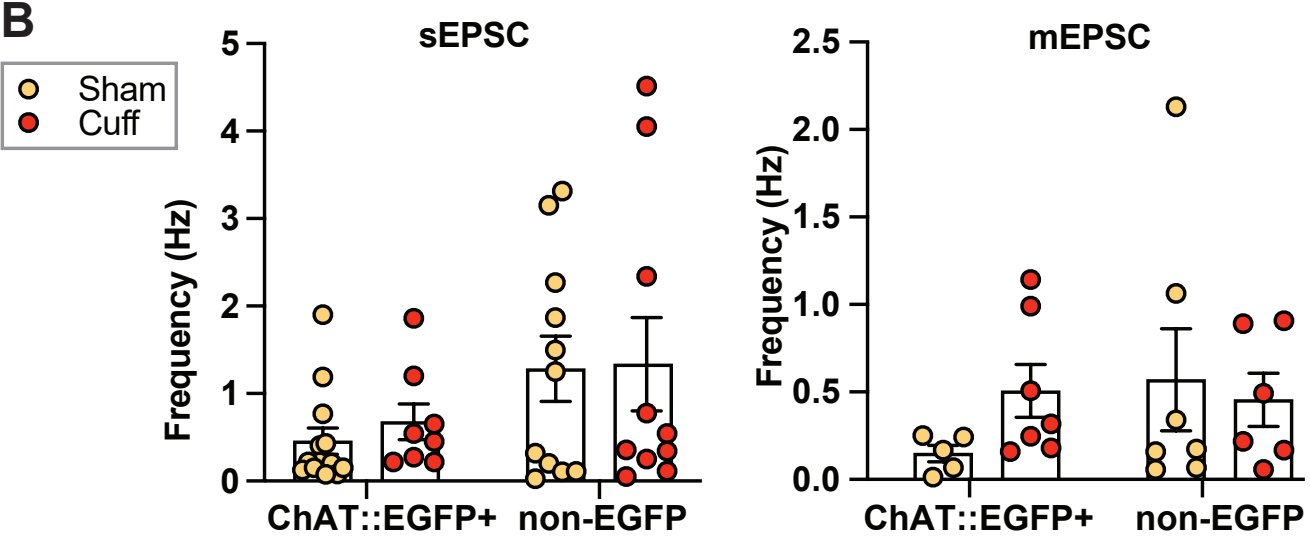

C
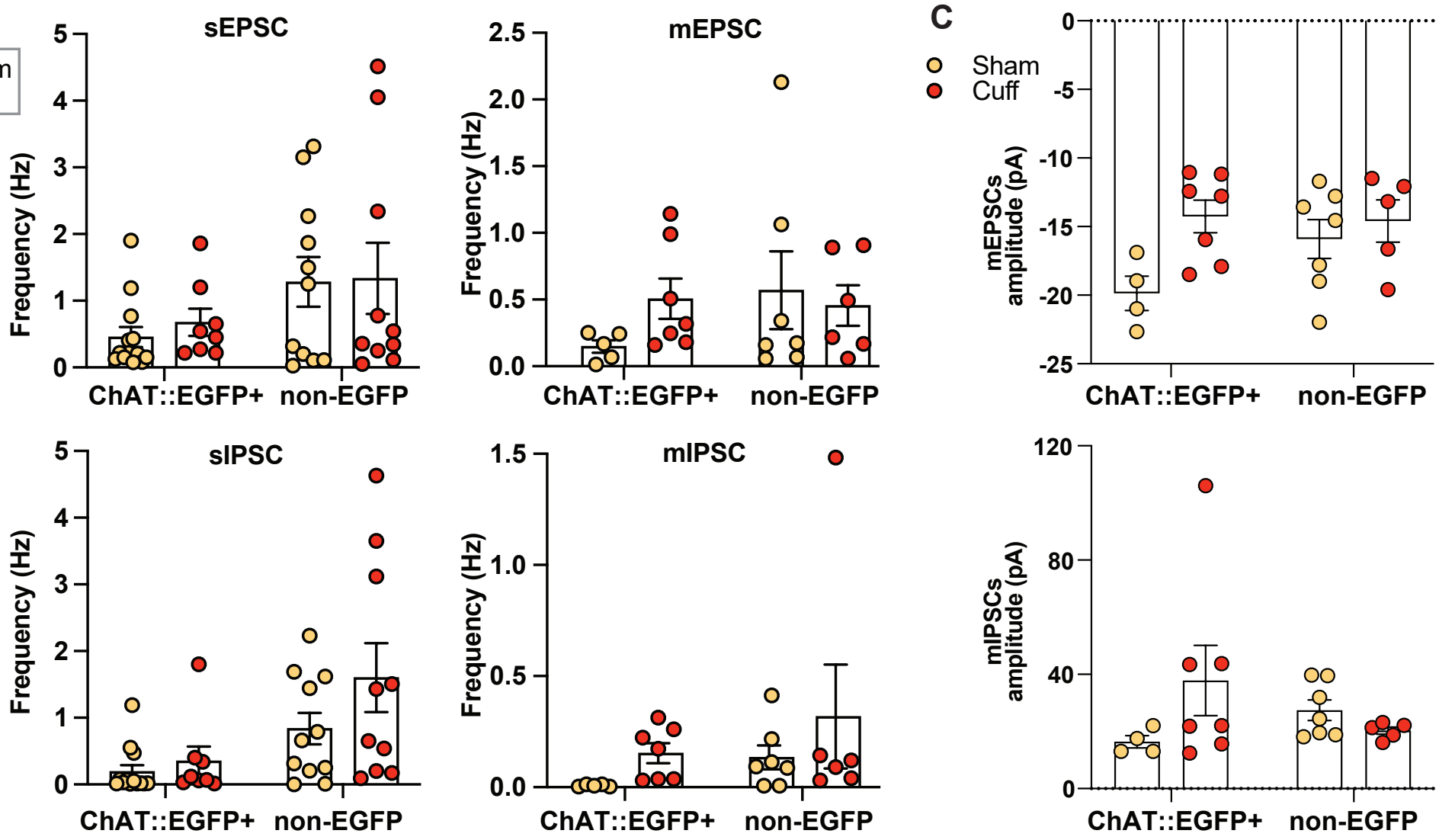

Figure 4: Number and synaptic inputs to Dorsal Horn cholinergic interneurons in sham and cuff mice A: Representative in vitro patch-clamp recordings from LIII/IV ChAT::EGFP (top) and non-EGFP (bottom) neurons, in Sham and Cuff mice. Recordings are performed at $0 \mathrm{mV}$ to record IPSCs and $-60 \mathrm{mV}$ to observe EPSCs. A1 : spontaneous excitatory (sEPSC) and inhibitory (sIPSC) postsynaptic currents, A2 : miniature mEPSC and mIPSC currents. B: Frequency of spontaneous (left) and miniature (right) EPSCs (top) and IPSCs (bottom) recorded in ChAT::EGFP and non-EGFP cells in horizontal slices of sham and cuff mice $(n=5-13$ neurons per group). The distribution of frequencies being non-normal, their $\log 10$ was taken to perform the ANOVA. There was no statistical difference in EPSCs frequencies between groups (ChAT::EGFP+ vs. non-EGFP, Sham vs. Cuff, spontaneous vs. miniature). For IPSCs, main effects were significant: neuron (ChAT::EGFP vs. non-EGFP, $p=0.000179$, 2-way ANOVA), surgery (sham vs. cuff, $\mathrm{p}=0.004616,2$-way ANOVA) and type of current (spontaneous vs. miniatures, $\mathrm{p}=1.051 \mathrm{e}-3,2$-way ANOVA).

C: Amplitude of miniature EPSC and IPSC of ChAT::EGFP+ and Non-EGFP+ neurons in sham and cuff mice $(n=4-7$ neurons per group). Data was normalized through inversion. There was no signicant difference between groups for mEPSCs (ANOVA). There was a significant Surgery-Neuron interaction for mIPSCs amplitude $(\mathrm{p}=0.0437)$ although this was not confirmed by post-hoc analysis. 


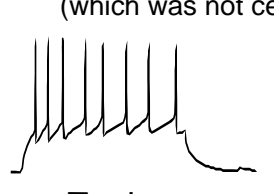

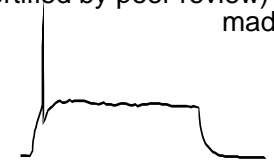

Single spike

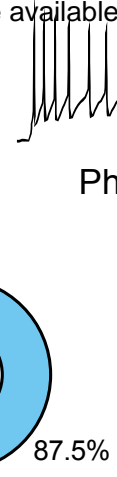

$20 \mathrm{mVL} \quad 20 \mathrm{mVL} / \mathrm{ms}$

Phasic

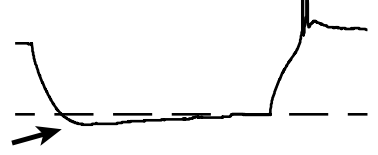

B
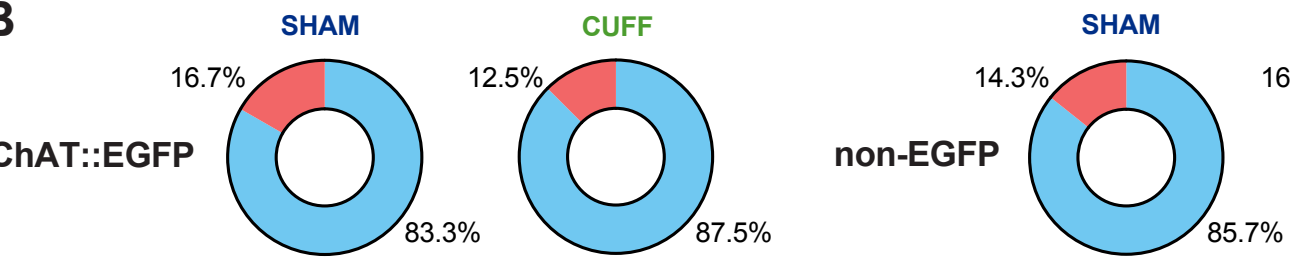

CUFF

C

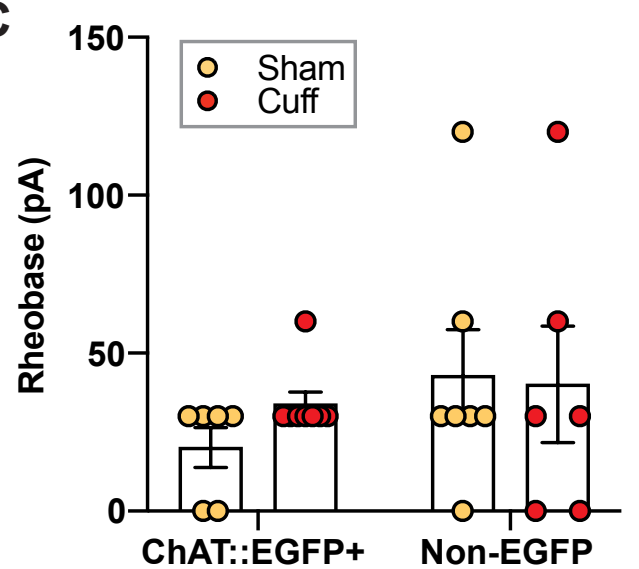

D

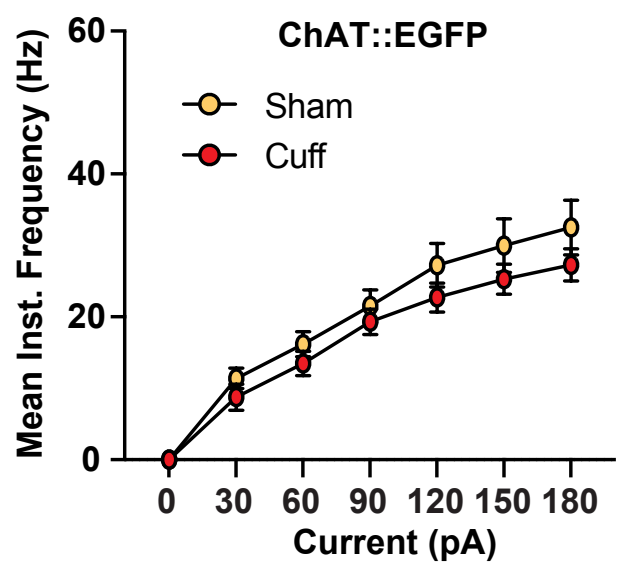

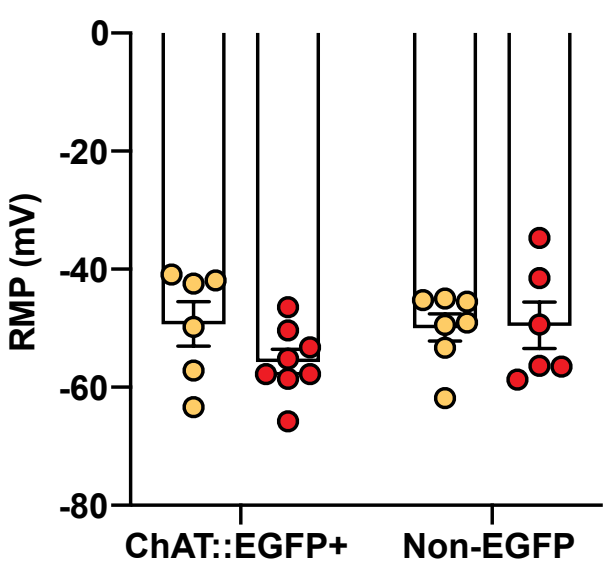

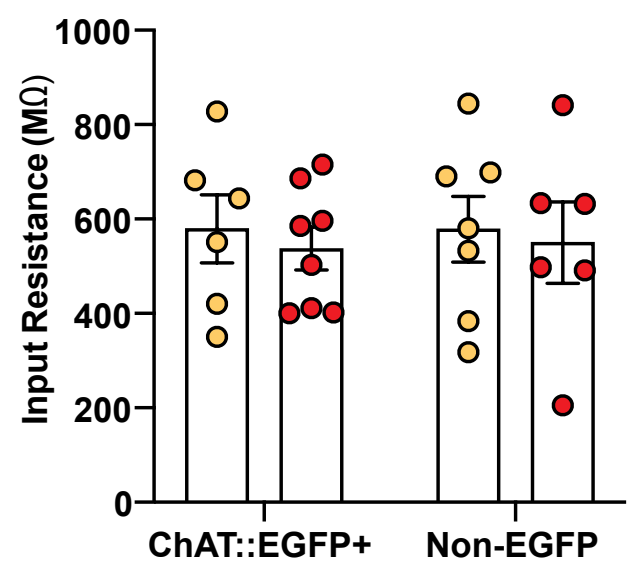

E
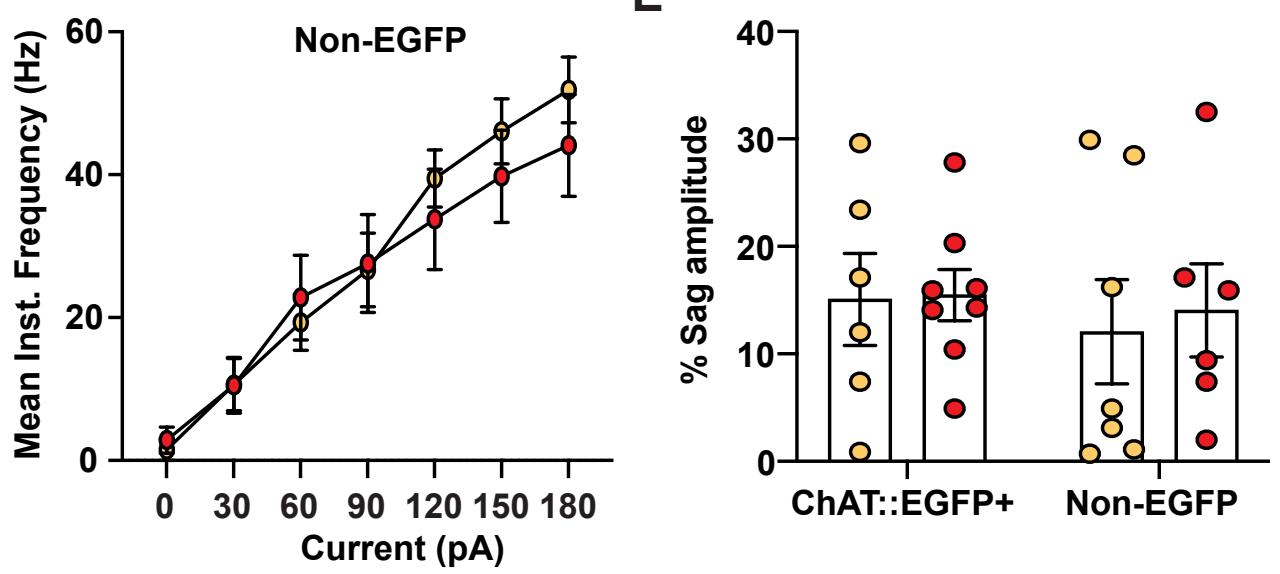

Tonic Firing Single Firing

Phasic Firing

Figure 5: Active and passive properties of lamine III/IV ChAT::EGFP+ and Non-EGFP cells in sham and cuff mice. A: (A1) Recording illustrating 3 different types of firing patterns, observed after injection of 90 pA depolarizing current: tonic, single-spike and phasic, (A2) Example of a response to a hyperpolarizing current showing post-inhibitory rebound. Arrow: hyperpolarizing "sag."

B: The distribution of different firing patterns is not different in the two neuronal populations or as a consequence of the surgery. Surgery-Neuron-Firing pattern interaction: $\mathrm{p}=0.6697,3 \times 3 \times 3$ contingency tables.

C: Electrophysiological properties of ChAT::EGFP and Non-EGFP cells (Group $n=6-8)$ : Rheobase $(3 \times 3 \times 3$ contingency tables, $p=0.3765)$, Resting membrane potential $[R M P](p=0.2706$, Surgery-Neuron interaction: $p=0.253)$ and Input resistance ( $p=0.9191,2$ way-ANOVA) were unaltered in both populations following neuropathy.

D: Mean Instantaneous frequencies of spikes in function of the injected current, as recorded in ChAT::EGFP and Non-EGFP neurons is similar in sham and cuff animals (Group $n=6-8$ neurons). There was a significant effect of neuron type (ChAT::EGFP vs. non-EGFP, $p=1.15 \mathrm{e}-2$, nparLD), current injected (between 0 - 180pA, $\mathrm{p}=3.91 \mathrm{e}-84$, nparLD) and a neuron:current injected interaction (comparing different injected currents in ChAT::EGFP and non-EGFP, $p=2.30 \mathrm{e}-2$, nparLD); details in suppl. Fig. 3. There was no Surgery-Neuron-depolarizing current interaction: $p=$ $7.53 \mathrm{e}-01, \mathrm{nparLD})$

E: Sag amplitude, expressed as a \% of the maximum hyperpolarization of the trace. There was no observable differences in the sag amplitudes (Group $\mathrm{n}=6-8,2$ way-ANOVA, $\mathrm{p}=0.8439$ ). 

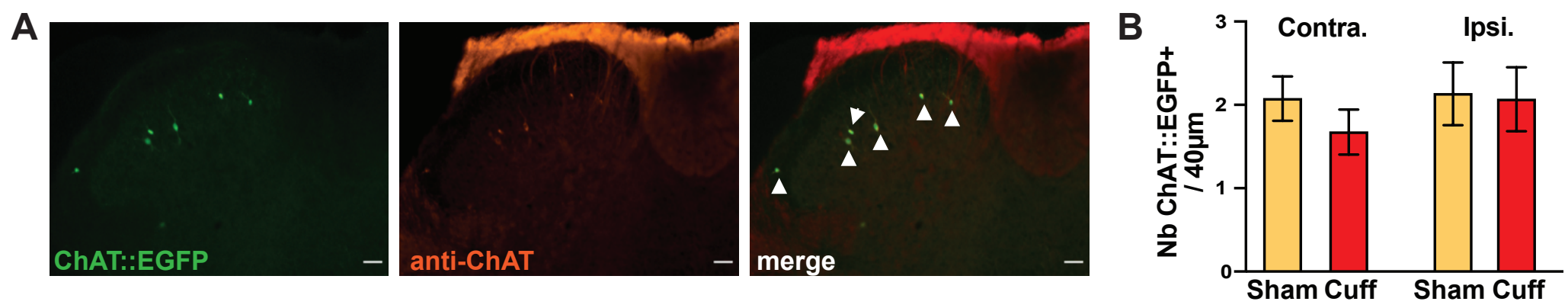

Figure 6: The number of DH cholinergic interneurons is unaltered after cuff surgery

A: Representative illustration of endogeous ChAT::EGFP and anti-ChAT labeling in the ipsilateral dorsal horn of a cuff mouse. The arrowhead denotes overlapping signals (scale $=100 \mu \mathrm{m})$.

B: Quantification of the total number of DH cholinergic interneurons (ChAT::EGFP fluorescence) in the lumbar (L3-L6) cord of sham and cuff mice [ $\mathrm{N}$ for Sham and Cuff $=2$ mice, 30 sections per condition, $60-64$ cells in total per condition]. There is no significant difference between groups (Contralateral side- Surgery-Staining interaction: $\mathrm{p}=0.329$; 2-way ANOVA $\mid$ Ipsilateral side- Surgery-Staining interaction: $\mathrm{p}>0.99$; 2-way ANOVA). 

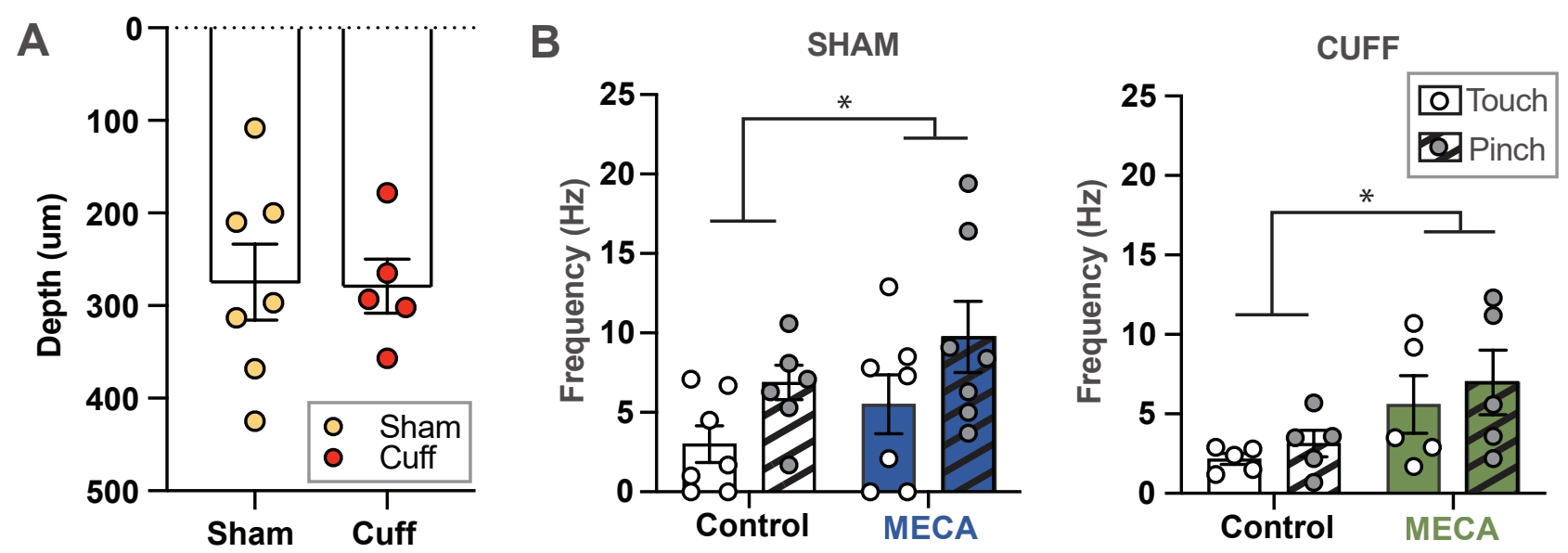

Figure 7: Touch and pinch responses in spinal dorsal horn neurons are modulated by endogenous acetylcholine via nicotinic receptors.

A: The depth of recorded DH neurons was similar in sham $(274.4 \pm 41.06 \mu \mathrm{m})$ and cuff $(279.0 \pm$ $29.33 \mu \mathrm{m}$ ) mice (sham vs cuff: $\mathrm{p}=0.9353$; unpaired t-test).

B: Mecamylamine increased the intensity of the response of spinal dorsal horn neurons to mechanical stimulation in sham $(\mathrm{N}=7$ neurons, Drug effect: $\mathrm{p}=0.0202$; repeated-measures 2 way ANOVA) and cuff mice ( $N=5$ neurons, Drug effect: $\mathrm{p}=0.0160$; repeated-measures 2 way ANOVA). 
